\title{
Dose-dependent glucosuria of DWP16001, a novel selective SGLT-2 inhibitor, in healthy subjects
}

\author{
Jungi Hwang ${ }^{1}$, SeungHwan Lee ${ }^{2}$, Wan Huh ${ }^{3}$, Jumi Han ${ }^{3}$, Jaeseong Oh², In-Jin Jang ${ }^{2}$, and \\ Kyung-Sang $\mathrm{Yu}^{2}$ \\ ${ }^{1}$ Chungbuk National University Hospital \\ ${ }^{2}$ Seoul National University College of Medicine \\ ${ }^{3}$ Daewoong Pharmaceutical Co Ltd
}

November 27, 2021

\begin{abstract}
DWP16001 is a novel sodium-glucose cotransporter-2 (SGLT2) inhibitor under development for the treatment of type 2 diabetes mellitus. This study was conducted to evaluate the pharmacokinetics (PK), pharmacodynamics (PD), and safety of DWP16001 after single and multiple doses in healthy subjects. A randomized, double-blind, placebo- and active-controlled, single- and multiple-dose study was conducted. Twelve subjects in each dose group received a single dose $(0.2,0.5,1.0,2.0$, or $5.0 \mathrm{mg})$ or multiple doses $(0.1,0.3,0.5,1.0$, or $2.0 \mathrm{mg}$ once daily for 15 consecutive days) of DWP16001, dapagliflozin $10 \mathrm{mg}$, or placebo, in a ratio of 8:2:2. Serial blood samples and interval urine samples were collected for PK and PD analyses. Safety and tolerability were assessed throughout the study period. A dose-dependent increase in urinary glucose excretion (UGE) was observed after a single dose, and the steady-state UGE was 50-60 g/day after multiple doses in the dose range of $0.3-2.0 \mathrm{mg}$. DWP16001 was rapidly absorbed with the time to peak plasma concentration of 1.0 - 3.0 hours, and eliminated with a mean elimination half-life of 13 - 29 hours. The systemic exposure of DWP16001 increased proportionally with the dose after multiple administrations in the range of $0.1-2.0 \mathrm{mg}$. DWP16001 was well tolerated in all dose groups. DWP16001 caused glucosuria in a dose-dependent manner, and systemic exposure was observed after multiple doses. DWP16001 was well tolerated up to $5.0 \mathrm{mg}$ after a single oral dose and up to $2.0 \mathrm{mg}$ after multiple oral administration
\end{abstract}

Dose-dependent glucosuria of DWP16001, a novel selective SGLT-2 inhibitor, in healthy subjects

Jun Gi Hwang ${ }^{1,2}$, SeungHwan Lee, ${ }^{1}$ Wan Huh $^{3}$, Jumi Han ${ }^{3}$, Jaeseong Oh$^{1}$, In-Jin Jang ${ }^{1}$, Kyung-Sang $\mathbf{Y u}^{1}$

${ }^{1}$ Department of Clinical Pharmacology and Therapeutics, Seoul National University College of Medicine and Hospital, Seoul, Republic of Korea

${ }^{2}$ Department of Clinical Pharmacology and Therapeutics, Chungbuk National University College of Medicine and Hospital, Chungcheongbuk-do, Republic of Korea

${ }^{3}$ Daewoong Pharmaceutical Co., Ltd., Seoul, Republic of Korea

* Correspondence: Kyung-Sang Yu

Department of Clinical Pharmacology and Therapeutics, Seoul National University College of Medicine and Hospital, 101 Daehak-ro, Jongno-gu, Seoul 03080, Republic of Korea

Tel +82 220722343

Fax +8227429252 
Email: ksyu@snu.ac.kr

Abstract

Aims

DWP16001 is a novel sodium-glucose cotransporter-2 (SGLT2) inhibitor under development for the treatment of type 2 diabetes mellitus. This study was conducted to evaluate the pharmacokinetics (PK), pharmacodynamics (PD), and safety of DWP16001 after single and multiple doses in healthy subjects.

\section{Methods}

A randomized, double-blind, placebo- and active-controlled, single- and multiple-dose study was conducted. Twelve subjects in each dose group received a single dose $(0.2,0.5,1.0,2.0$, or $5.0 \mathrm{mg})$ or multiple doses $(0.1$, $0.3,0.5,1.0$, or $2.0 \mathrm{mg}$ once daily for 15 consecutive days) of DWP16001, dapagliflozin $10 \mathrm{mg}$, or placebo, in a ratio of 8:2:2. Serial blood samples and interval urine samples were collected for PK and PD analyses. Safety and tolerability were assessed throughout the study period.

\section{Results}

A dose-dependent increase in urinary glucose excretion (UGE) was observed after a single dose, and the steady-state UGE was 50-60 g/day after multiple doses in the dose range of $0.3-2.0 \mathrm{mg}$. DWP16001 was rapidly absorbed with the time to peak plasma concentration of $1.0-3.0$ hours, and eliminated with a mean elimination half-life of 13 - 29 hours. The systemic exposure of DWP16001 increased proportionally with the dose after multiple administrations in the range of $0.1-2.0 \mathrm{mg}$. DWP16001 was well tolerated in all dose groups.

\section{Conclusion}

DWP16001 caused glucosuria in a dose-dependent manner, and systemic exposure was observed after multiple doses. DWP16001 was well tolerated up to $5.0 \mathrm{mg}$ after a single oral dose and up to $2.0 \mathrm{mg}$ after multiple oral administrations.

\section{What is already known about this subject :}

Type 2 diabetes is the chronic metabolic disorder which prevalence has been increasing worldwide.

SGLT-2 inhibitor, the antidiabetic agent which inhibits reabsorption of glucose in the renal tubule, recommended in T2DM accompanied by cardiovascular disease.

\section{What this study adds :}

We studied the pharmacodynamics and pharmacokinetics of DWP16001, a novel SGLT-2 inhibitor under development.

This study proved the effect of DWP16001 as a potent SGLT-2 inhibitor. This finding may provide information for clinical research and combination with other antidiabetic agents.

\section{Introduction}

Type 2 diabetes mellitus (T2DM) is a major chronic metabolic disorder. Over 463 million people were diagnosed with T2DM in 2019 and the prevalence of T2DM has been increasing steadily worldwide [1]. T2DM is characterized by insulin insensitivity, resistance, and secretion defects caused by pancreatic beta cell dysfunction [2]. Metformin is the preferred initial pharmacologic agent for the treatment of T2DM[3,4]. However, sulfonylureas, thiazolidinedione, DPP-4 inhibitors, GLP-1 receptor agonists, and SGLT-2 inhibitors are prescribed as add-on drugs when target HbA1c levels are not achieved with metformin therapy $[5,6]$. Among these, SGLT-2 inhibitor is recommended in T2DM accompanied by cardiovascular disease such as heart failure [7]. 
Sodium glucose cotransporters (SGLT) are of two types. SGLT-1 is distributed throughout organs such as the intestine and kidneys and has low capacity and high affinity. SGLT-2 is primarily present in the kidneys and has a relatively high capacity and low affinity. Under normal physiological conditions, both transporters reabsorb glucose in the renal proximal tubule $(<180 \mathrm{~g} /$ day $)$, but the glucose uptake of SGLT-2 is over $97 \%$ in the renal proximal tubule $[8,9]$. When SGLT-2 is dysfunctional - as a result of mutations in the SLC5A2 gene - the renal capacity for absorbing glucose decreases to the residual capacity of SGLT-1 (80 g/day) [10].

SGLT-2 inhibitors are considered potential strategies for T2DM treatment because of the following advantages: (1) the mechanism of action of SGLT-2 inhibitors is independent of insulin [11]; (2) SGLT-2 inhibitors lower the risk of cardiovascular disease - a major complication of T2DM [12,13]; (3) SGLT-2 inhibitors protect kidney function by decreasing glomerular filtration rate (GFR) reversibly and preserving GFR in the long-term in patients with T2DM [14]; (4) SGLT-2 inhibitors promote weight loss, which lowers the risk of complications related to T2DM and can compensate for the weight gain that is a side effect of insulin and sulfonylureas [15]. Canagliflozin (Invokana ${ }^{\circledR}$ ), dapagliflozin (Farxiga ${ }^{\circledR}$ ), empagliflozin (Jardiance ${ }^{\circledR}$ ), and ertugliflozin (Steglatro ${ }^{\mathrm{TM}}$ ) have recently been approved by the US FDA and EMA for the treatment of T2DM [16].

DWP16001 is a novel SGLT-2 selective inhibitor under clinical development. In an in vitro study, DWP16001 selectively inhibited SGLT2 and showed high potency for SGLT2 inhibition - more than 1000 times that of SGLT1. DWP16001 is mainly metabolized by CYP3A4 and CYP2C19 into active metabolites M1 and M2 or via glucuronidation by UGT2B7, UGT1A4, and UGT1A9 [17]. The inhibitory potency of these metabolites for SGLT-2 was 10 times weaker than that of parent molecule. [18].

The purpose of this study was to evaluate the safety, pharmacokinetics (PKs), and pharmacodynamics (PDs) of DWP16001 after single and multiple doses in healthy Korean subjects.

\section{Methods}

\section{Subjects}

Prior to any study-related procedures, the subjects provided written informed consent during the screening period. Healthy Korean men aged 19 to 50 years, with a body mass index of $18-27 \mathrm{~kg} / \mathrm{m}^{2}$ were selected. Subjects were excluded from the study if they had a clinically significant history or suffered from a concurrent disease that could have affected the safety, PK, or PD of DWP16001. Exclusion criteria also included a GFR under $<90 \mathrm{~mL} / \mathrm{min}$ as calculated from the MDRD equation, AST or ALT $>1.5$ times the upper limit of normal, fasting serum glucose $>110 \mathrm{mg} / \mathrm{dL}$ or $<70 \mathrm{mg} / \mathrm{dL}$, or a glycosylated hemoglobin A1c (HbA1c) level over $6.5 \%$.

\section{Study Design}

This randomized, double-blind, active- and placebo-controlled, single- and multiple-dose study was conducted at the Seoul National University Hospital Clinical Trials Center in accordance with the Declaration of Helsinki and the rules of Korean Good Clinical Practice. The study protocol was reviewed by the Ministry of Food and Drug Safety (ClinicalTrials.gov: NCT03364985).

Twelve subjects in each dose group were orally administered a single dose or multiple doses for 15 consecutive days of DWP16001, dapagliflozin $10 \mathrm{mg}$ (active comparator), or placebo in a ratio of 8:2:2. The investigated dose levels were $0.2,0.5,1.0,2.0$ and $5.0 \mathrm{mg}$ in the single-dose arm, and $0.1,0.3,0.5,1.0$ and $2.0 \mathrm{mg}$ in the multiple-dose arm. Meals were provided 2, 6, and 10 hours post-dose; breakfast was not provided on the days of intensive pharmacokinetic evaluation or oral glucose tolerance test. In addition, the food effect was explored in the $2.0 \mathrm{mg}$ dose group in the single dose arm in a crossover manner. The subjects in this arm received the study drugs in a fasted state and received them once again in a fed state after a two-week 
washout. In the fed status, a high-fat meal of $900 \mathrm{kcal}$ was provided at 30 minutes prior to study drug administration.

\section{Pharmacodynamic (PD) evaluation}

In the single-dose arm, urine samples for urinary glucose excretion (UGE) analysis were collected up to 168 h (collection interval: $0-4,4-8,8-12,12-24,24-48,48-72,72-96,96-120,120-144$, and $144-168 \mathrm{~h}$ postdose). In the multiple-dose arm, urine collection was conducted at Day 1 and Day 15 at the same time intervals as the single-dose arm. Urine samples for PD analysis were separated in a $10 \mathrm{~mL}$ conical tube and stored at $-20 \operatorname{deg} \mathrm{C}$ until analysis. Urine glucose was measured using an automated analyzer (TBA-120FR; Toshiba Medical Systems, Tochigi, Japan). Urine PD parameters were defined as UGE from 0 to the last measurement $\left(\mathrm{UGE}_{\text {last }}\right)$ and $\mathrm{UGE}$ within 24 hours after the first and last dose $\left(\mathrm{UGE}_{0-24 \mathrm{~h}}, \mathrm{UGE}_{15 \mathrm{~d}}\right)$.

An oral glucose tolerance test (75 g OGTT) was performed in the multiple-dose period before administration as the baseline (Day -2) and during the steady state (Day 14). Blood samples for serum glucose were obtained at pre-dose and $0.5,1,1.5,2,3$, and 4 hours post-dose on those days. Plasma insulin concentration samples were collected for up to 6 hours on the same day as serum glucose. Each blood sample collected in a serum separator tube was temporarily left at room temperature for approximately 30 minutes, and then centrifuged for 10 minutes at $1820 \mathrm{x} g$. The supernatant was collected in Eppendorf tubes and stored at -20 $\operatorname{deg} \mathrm{C}$ until analysis. Serum concentrations of glucose were measured using an automated analyzer (automatic chemical analyzer: TBA-FX8, Toshiba, Japan). The plasma concentrations of insulin were analyzed with the immunoradiometric assay (IRMA) method (gamma counter: Dream Gamma-10, Shin Jin, Republic of Korea)

The maximum serum glucose concentration $\left(\mathrm{E}_{\max }\right)$ was determined from the individual profiles, and the area under the 4-hour glucose-time curve $\left(\mathrm{AUEC}_{0-4 \mathrm{~h}}\right)$ was calculated.

\section{Pharmacokinetic (PK) evaluation}

Blood samples for single-dose PK evaluation were obtained pre-dose and $0.25,0.5,0.75,1,1.5,2,3,4,6$, $8,10,12,24,36,48,72$, and 96 hours after administration. In the multiple dose arm, blood samples were obtained up to 24 and 96 hours post-dose after the first and last administration (Day 1 and Day 15) in a manner equal to that of the single-dose PK. In addition, pre-dose PK samplings (Day 1, Day 2, Day 3, Day 4, Day 5, Day 7, Day 10, Day 13, and Day 15) were conducted to check whether the steady-state was reached. At each blood sampling point, $12 \mathrm{~mL}$ of blood was collected in a sodium heparin tube and centrifuged for 10 min at $1820 \mathrm{~g}$. The supernatant was separated in Eppendorf tubes and stored at -70 degC until analysis.

Urine samples for PK evaluation were also collected up to 96 hours post-dose after a single dose in the single-dose arm. In the multiple-dose arm, urine samples were collected up to 24 hours after the first dose (Day 1) and up to 96 hours post-dose after the last dose (Day 15). Urine samples were separated and stored in the same way as the plasma samples.

The plasma concentrations of DWP16001 and metabolites were determined using high-performance liquid chromatography coupled with tandem mass spectrometry (LC-MS/MS) using a SCIEX API 5000 system (SCIEX, Framingham, MA, USA) in positive-ion electrospray mode. The concentration of the parent molecules and metabolites in the urine was measured using LC-MS/MS in negative-ion electrospray mode. The lower limits of quantification of DWP16001 in the plasma and urine were $0.1 \mu \mathrm{g} / \mathrm{L}$ and $0.05 \mu \mathrm{g} / \mathrm{L}$, respectively. The corresponding values of M1 and M2 were 0.04 and $0.05 \mu \mathrm{g} / \mathrm{L}$ in plasma, and 0.04 and 0.1 $\mu \mathrm{g} / \mathrm{L}$ in urine, respectively.

Single-dose and multiple-dose pharmacokinetic parameters of DWP16001 and its metabolites were determined using a noncompartmental method with Phoenix ${ }^{\circledR}$ WinNonlin ${ }^{\circledR} 7.1$ (Certara, L.P., St. Louis, MO, USA). The PK parameters of DWP16001 and M1/M2 included the maximum plasma concentration $\left(\mathrm{C}_{\max }\right)$ and time to reach $\mathrm{C}_{\max }\left(\mathrm{T}_{\max }\right)$ and were directly determined from the observed individual plasma 
concentration-time profiles. The terminal elimination half-life $\left(t_{1 / 2}\right)$ was calculated as the natural logarithm of 2 divided by $\lambda_{z}$, which is the terminal elimination rate constant estimated in the linear portion of the decline of the natural logarithmic-transformed individual plasma concentrations. Areas under the concentrationtime curve $\left(\mathrm{AUC}_{\text {last }}, \mathrm{AUC}_{\text {inf }}\right.$ and $\left.\mathrm{AUC}_{\text {tau }}\right)$ were calculated using the linear-up log-down trapezoidal rules. Apparent clearance $(\mathrm{CL} / \mathrm{F}$ ) was derived as the administered dose divided by the AUC. The renal clearance $\left(\mathrm{CL}_{\mathrm{R}}\right)$ and the fraction of the dose excreted into the urine $(\mathrm{fe})$ were calculated through urine analysis. The accumulation ratio $(\mathrm{R})$ was calculated as the ratio of $\mathrm{AUC}_{\text {tau }}$ after the last dose after multiple doses (Day 15) to that after a single dose (Day 1 ). The metabolic ratio (MR) was determined as the ratio of the $\mathrm{AUC}_{\text {tau }}$ of the metabolites to that of the parent molecules after multiple administrations.

\section{PK-PD Relationship}

The $\mathrm{AUC}_{\text {tau }}$ after a single dose in both the single dose arm and multiple dose arm, and the $\mathrm{UGE}_{0-24 \mathrm{~h}}$ were selected as PK and PD parameters, respectively. A sigmoid $\mathrm{E}_{\max }$ model was selected to show the relationship, and half of the maximum effect $\left(\mathrm{EC}_{50}\right)$, maximum effect $\left(\mathrm{E}_{\max }\right)$, and Hill coefficient $(\gamma)$ were calculated using the following equation:

$$
E=\frac{E_{\max } \bullet C^{r}}{\mathrm{EC}_{50}{ }^{r}+C^{r}}
$$

Spearman's correlation coefficients and p-values were calculated to assess the PK/PD relationship.

\section{Safety Evaluation}

Safety was assessed based on adverse events (AEs), vital signs, physical examinations, electrocardiograms, and clinical laboratory tests, including urinalysis, throughout the study. The severity of the AE was classified as mild, moderate, or severe, and the causal relationship of the AE was evaluated to determine whether the $\mathrm{AE}$ was related or not to DWP16001. An adverse drug reaction (ADR) was defined as an AE that could not be ruled out as unrelated to DWP16001.

N-acetyl-b-D-glucosaminidase (NAG) and beta 2-microglobulin (B2M) levels were measured at baseline (Day -1) and steady-state (Day 15) to evaluate the extent of proximal tubule (SGLT-2 receptor -presented) damage as exploratory safety markers. Urine NAG and B2M were measured using commercial radioimmunoassay kits (Beckman Coulter, Fullerton, CA, USA).

\section{Statistical analysis}

Statistical analyses were performed using SAS 9.4 software (SAS Institute, Inc., Cary, NC, USA). All descriptive data were summarized as the mean and standard deviation for continuous variables, and frequencies and percentages for categorical data. With regard to the $\mathrm{C}_{\max }$ and $\mathrm{AUC}$ of DWP16001, dose proportionality in the plasma was evaluated through power model analysis using SAS 9.4 software. To compare the pharmacodynamic effect between DWP16001 and dapagliflozin, geometric mean ratios (GMRs, DWP16001 to dapagliflozin) and the 2-sided $90 \%$ confidence interval (CIs) of $\mathrm{UGE}_{0-15 \mathrm{~d}}$ were calculated. The effect of food was assessed through the GMR and 90\% CIs of the PK parameters (log-transformed $\mathrm{C}_{\max }$ and AUC) and $\mathrm{PD}$ parameter $\left(\mathrm{UGE}_{0-24 \mathrm{~h}}\right)$ were calculated. The incidences of AEs and ADRs were compared among the treatment groups using the Kruskal-Wallis test. 


\section{Results}

\section{Demographics}

In the single-dose arm, a total of 61 subjects were enrolled, and 60 subjects completed the study. The mean ( \pm standard deviation) age, body weight and BMI of the randomized subjects were $30.75 \pm 6.89$ years, 71.06 $\pm 7.48 \mathrm{~kg}$, and $23.43 \pm 2.06 \mathrm{~kg} / \mathrm{m}^{2}$, respectively.

In the multiple-dose arm, 62 subjects were enrolled, and 60 subjects completed the study. The mean $( \pm$ standard deviation) age, body weight and BMI of the randomized subjects were $30.84 \pm 5.43$ years, 70.72 $\pm 7.15 \mathrm{~kg}$, and $23.47 \pm 2.07 \mathrm{~kg} / \mathrm{m}^{2}$, respectively. The demographic characteristics were comparable among the dose groups for both single and multiple arms.

\section{Pharmacodynamics}

In the single dose arm, $\mathrm{UGE}_{0-24 \mathrm{~h}}$ and $\mathrm{UGE}_{\text {last }}$ increased in a dose-dependent manner in the range of 0.2 to $1.0 \mathrm{mg}$. $\mathrm{UGE}_{0-24 \mathrm{~h}}$ tended to be saturated in the range of $1.0 \mathrm{mg}$ to $5.0 \mathrm{mg}$ of DWP16001, which was similar to that of dapagliflozin $10 \mathrm{mg}$. Meanwhile, $\mathrm{UGE}_{\text {last }}$ was higher over $1.0 \mathrm{mg}$ of DWP16001 than that of dapagliflozin (Figure 1,2 Table 1).

In the multiple dose arm, daily UGE reached a plateau at 50 - $60 \mathrm{~g}$ /day at the steady state. The UGE was higher with all doses of DWP16001 and dapagliflozin $10 \mathrm{mg}$ compared with placebo (Table S1). The mean UGE over 24 hours at steady state of $0.1 \mathrm{mg}, 0.3 \mathrm{mg}, 0.5 \mathrm{mg}, 1.0 \mathrm{mg}$, and $2.0 \mathrm{mg}$ DWP16001 were $36.1 \mathrm{~g}$, $48.3 \mathrm{~g}, 55.1 \mathrm{~g}, 56.8 \mathrm{~g}$, and $53.4 \mathrm{~g}$, respectively, and the corresponding value of dapagliflozin $10 \mathrm{mg}$ was 40.5 g (Figure 1,2, Table 1).

In the OGTT after dosing for 14 consecutive days of DWP16001, the $\mathrm{E}_{\max }$ of the plasma glucose was lower than that of baseline. The mean $\mathrm{E}_{\max }$ changed from baseline of $0.1 \mathrm{mg}, 0.3 \mathrm{mg}, 0.5 \mathrm{mg}, 1.0 \mathrm{mg}$ and $2.0 \mathrm{mg}$ DWP16001 were $-27.5,-32.8,-7.8,-23.9$ and $-17.4 \mathrm{mg} / \mathrm{dL}$, respectively. These values were different from -2.2 $\mathrm{mg} / \mathrm{dL}$ of placebo, but similar to $-21.6 \mathrm{mg} / \mathrm{dL}$ of dapagliflozin $10 \mathrm{mg}$. A similar pattern was observed for AUEC $_{0-4 \mathrm{~h}}$ after OGTT. The mean AUEC $_{0-4 \mathrm{~h}}$ changed from baseline was positive in placebo $(4.3 \mathrm{mg} * \mathrm{~h} / \mathrm{dL})$ , whereas $0.1 \mathrm{mg}, 0.3 \mathrm{mg}, 0.5 \mathrm{mg}, 1.0 \mathrm{mg}, 2.0 \mathrm{mg}$ DWP16001 were -46.8 , -55.6, -40.1, -54.0 and -21.9 $\mathrm{mg}^{*} \mathrm{~h} / \mathrm{dL}$, respectively. Postprandial insulin showed a decreasing tendency after multiple doses, but it was not statistically significant (Figure S1, Table S2).

\section{Pharmacokinetics}

DWP16001 was rapidly absorbed after a single dose with $\mathrm{T}_{\max }$ between 1 and $3 \mathrm{~h}$ post-dose. After reached a $\mathrm{T}_{\max }$, plasma concentration of DWP16001 fell in a biphasic manner with the elimination half-life of 11 to 28 hours (Figure 3 ). The $t_{1 / 2}$ was tended to decrease as the dose of DWP16001 increased (Table 2).

After multiple doses, the time-plasma concentration was similar to that of the single-dose arm. The mean $\mathrm{T}_{\max }$ and $\mathrm{t}_{1 / 2}$ at steady state were within a range of 1 to 2 hours and 17 to 23 hours, respectively. After multiple administrations, the $95 \%$ CIs of the slope of the log-transformed $\mathrm{C}_{\mathrm{max}, \mathrm{ss}}$ and $\mathrm{AUC}_{\text {tau,ss }}$ included 1.0 (0.98-1.10 and 0.98-1.09, respectively), thus considered dose-proportional. The mean accumulation index was approximately 1.6 to 1.9 over the range of $0.1 \mathrm{mg}$ to $2.0 \mathrm{mg}$ of DWP16001. Less than $3 \%$ of DWP16001 was excreted unchanged in the urine at all doses (Table 2). The metabolic ratio of M1 ranged from 0.20 to 0.25. M2 was detected in very low amounts compared to the parent molecules in all dose groups (Table S3).

\section{PK-PD relationship}

Drug exposure and UGE showed a clear exposure-response relationship. The $\mathrm{AUC}_{\text {tau }}$ and UGE over $24 \mathrm{~h}$ after the last dose were correlated, with a Spearman correlation coefficient of $0.4061(\mathrm{p}=0.0002)$. The PK- 
PD relationship was well-explained by a sigmoid $\mathrm{E}_{\max }$ model, and the $\mathrm{E}_{0}, \mathrm{E}_{\max }, \mathrm{EC}_{50}, \mathrm{EC}_{90}$, and $\gamma$ were calculated to be $0.1 \mathrm{~g}, 58.8 \mathrm{~g}, 31.8 \mu \mathrm{g} \cdot \mathrm{h} / \mathrm{L}, 58.4 \mu \mathrm{g} \cdot \mathrm{h} / \mathrm{L}$, and 3.6, respectively (Figure 4).

\section{Effect of Food}

After a high-fat meal was provided, the median $\mathrm{T}_{\max }$ was prolonged from 1.3 to 2.5 hours, and the $\mathrm{C}_{\max }$ was decreased approximately $43 \%$ (GMR [90\% CIs]: 0.57 [0.45-0.72]), compared to that of fasting state (Figure 5). However, the $\mathrm{AUC}_{\text {inf }}$ was similar between fed and fasting state (GMR [90\% CIs]: 0.97 [0.82-1.16]). The UGE was comparable between fed and fasting state; GMR [90\% CIs] of $\mathrm{UGE}_{0-24 \mathrm{~h}}$ and $\mathrm{UGE}_{\text {last }}$ were 0.80 [0.66-0.97] and $0.90[0.68-1.19]$, respectively.

\section{Safety and Tolerability}

DWP16001 was found to be safe and tolerable in all dose groups, both in single- and multiple-dose administration. In the single-dose arm, treatment-emergent adverse events (TEAEs) were reported in 12 cases by 5 subjects who received DWP16001. In the multiple-dose arm, 27 cases were reported in 13 subjects who received DWP16001, and four cases were reported in three subjects who received placebo. Adverse drug reactions (ADRs) that were determined to have a causal relationship with the investigational product were reported in two cases from one subject in the single-dose group and 22 cases from 12 subjects in the multiple-dose group (Table 3). The most frequently reported ADRs were abdominal pain, headache, and rhinorrhea. Abdominal pain was reported by three subjects (1 in the $0.1 \mathrm{mg}$ group and 2 in the $1.0 \mathrm{mg}$ group). All TEAEs were mild and spontaneously resolved. There were no serious adverse events, and no subject withdrew from the study owing to adverse events. No other clinically significant changes were observed in the clinical laboratory tests, vital signs, ECGs, or physical examinations.

Renal tubular marker [N-acetyl- $\beta$-D-glucosaminidase (NAG) and $\beta 2$-microglobulin (B2M)] tests were conducted in the multiple-dose arm. Only one subject (DWP16001 $2.0 \mathrm{mg}$ group) had an increased B2M by more than $0.5 \mu \mathrm{g} / \mathrm{mL}$ compared to the baseline, but it was found to be closer to the baseline level in a post-study visit. The mean change in NAG and B2M concentrations showed no significant increase across the different dose groups (Table S4).

\section{Discussion}

In this study, the amount of UGE showed maximum values of $>60 \mathrm{~g}$ /day in groups treated $1.0 \mathrm{mg}$ or higher after a single dose. The steady state daily UGE seemed to peak at $>50 \mathrm{~g} /$ day in the groups treated with $0.5 \mathrm{mg}$ or higher after multiple dosing. The UGE over $24 \mathrm{~h}$ seemed to be saturated at higher doses. This was considered to be compensation for glucose reabsorption in the proximal tubule by the SGLT1 receptor under normoglycemic conditions and by a decrease in renal clearance $[19,20]$.

In previous clinical studies of SGLT-2 inhibitors in healthy subjects, the UGE after multiple doses was 33.8 $\mathrm{g} /$ day with canagliflozin $100 \mathrm{mg}$ and $35.5 \mathrm{~g}$ /day with dapagliflozin $10 \mathrm{mg}$ [20,21]. The corresponding values for canagliflozin $100 \mathrm{mg}$ and dapagliflozin $10 \mathrm{mg}$ after multiple doses in T2DM patients both exceeded 70 $\mathrm{g} /$ day and were demonstrated to show clinical efficacy $[22,23]$. Considering this relationship between the results in healthy subjects and patients, DWP16001 doses above $0.1 \mathrm{mg}$ are expected to show clinical efficacy in T2DM patients.

Urine glucosuria induced by administration of DWP16001 lasted up to $168 \mathrm{~h}$ after a single dose. DWP16001 maintained a longer inhibition of SGLT2 than dapagliflozin and ipragliflozin in an in vitro test [18]. In addition, DWP16001 showed a better kidney distribution and longer elimination half-life than dapagliflozin and ipragliflozin at the ICR mouse and these results could have accounted for the long duration of UGE of DWP16001 [18]. Considering the elimination half-life and the usage of most type2 DM treatments (daily medication), DWP16001 seems to be desirable to be administered as a once daily therapy. Additionally, 
it is expected that long-lasting UGE and anti-hyperglycemic effect suppress glucose excursion to achieve desirable treatment target even in T2DM patients with low medication adherence.

The $\mathrm{C}_{\max }$ and $\mathrm{AUC}_{\mathrm{tau}}$ of DWP16001 after multiple administrations showed dose-proportional increments in the range of $0.1 \mathrm{mg}$ to $2.0 \mathrm{mg}$. Meanwhile, the $t_{1 / 2}$ and $\mathrm{T}_{\max }$ were independent of the dose. A high-fat meal delayed the absorption of DWP16001 and decreased $\mathrm{C}_{\max }$, but drug exposure was almost unaffected. The fraction of DWP16001 excreted in an unchanged form in the urine was less than $2.5 \%$. This finding is consistent with nonclinical data that show that DWP16001 is extensively metabolized and mainly eliminated through a non-renal pathway in humans [17]. SGLT2 inhibitors have controversial effects on kidney injuries, but the PK characteristics of DWP16001 might be less affected by renal impairment. Further clinical pharmacology studies in subjects with decreased renal function will be needed to elucidate the PK characteristics of DWP16001.

The possibility of DWP16001-related kidney damage is expected to be relatively low considering the results obtained in healthy subjects. There are positive reports that the long-term use of SGLT-2 inhibitors decreases the burden on the glomerulus and shows a renoprotective effect $[25,26,27]$. In contrast, there is a suspicion that long-term use of SGLT2 inhibitors could lead to tubular injury depending on the mechanism of action $[28,29]$. This has been observed through the elevation of renal tubular markers in some previous studies $[30,31]$. Therefore, the levels of NAG and B2M were monitored in this study throughout the multiple dose period. After administration of $0.1 \mathrm{mg}$ DWP16001, NAG levels slightly increased at steady state compared to the baseline. However, this tendency was not dose-related, and the values returned to the respective baseline level during follow-up visits. B2M levels were not statistically different at all doses, including those in the placebo. Likewise, there was a similar pattern of the estimated GFR between the baseline and post-dose.

DWP16001 was generally safe and well tolerated among all doses in this study. The incidence of AEs after single and multiple doses of DWP16001 did not appear to be dose-related in either study. Genitourinary infection is known to be a major adverse event following the use of SGLT2 inhibitors [32], but only one subject in the $1.0 \mathrm{mg}$ multiple DWP16001 dose group suffered balanoposthitis; there were no other occurrences of genitourinary infections. In addition, only a few subjects experienced gastrointestinal adverse events, reflecting the inhibition of SGLT1 in the GI tract. This can be explained by the selective inhibitory effect of DWP16001 on SGLT2.

\section{Conclusions}

In summary, DWP16001 showed clear dose- and exposure-response relationships, exhibited linear PK characteristics and was well-tolerated in healthy subjects. These findings support further evaluation of DWP16001 as a treatment option for T2DM.

\section{Acknowledgments}

The authors certify that this manuscript represents valid work and that neither this manuscriptnor one with substantially similar content has been published or is being considered for publication elsewhere. Part of the data were previously partially presented at the 55th European association for the study of diabetes (EASD) Annual Meeting, 16-20 September 2019. The data that support the findings of this study are available from the corresponding author, Kyung-Sang Yu, upon reasonable request. This study was funded by Daewoong Pharmaceutical Co., Ltd. 


\section{Conflicts of Interest}

Wan Huh and Jumi Han are employees of Daewoong Pharmaceutical Co., Ltd. The other authors report no conflicts of interest.

\section{References}

1. Saeedi, P., Petersohn, I., Salpea, P., Malanda, B., Karuranga, S., Unwin, N., .. \& \& IDF Diabetes Atlas Committee. (2019). Global and regional diabetes prevalence estimates for 2019 and projections for 2030 and 2045: Results from the International Diabetes Federation Diabetes Atlas. Diabetes research and clinical practice, 157, 107843.

2. Maitra A, Abbas AK. Endocrine system. In: Kumar V, Fausto N, Abbas AK (eds). Robbins and Cotran Pathologic basis of disease (7th ed) 2005. Philadelphia, Saunders; 1156-1226.

3. Neumiller, Joshua J., and Guillermo E. Umpierrez. "2018 Standards of Care Update: Pharmacologic Approaches to Glycemic Management in People with Type 2 Diabetes." Diabetes Spectrum 31.3 (2018): 254-260.

4. American Diabetes Association. "9. Pharmacologic Approaches to Glycemic Treatment: Standards of Medical Care in Diabetes-2020." Diabetes Care 43.Suppl 1 (2020): S98.

5. Dash, Ranjeet Prasad, R. Jayachandra Babu, and Nuggehally R. Srinivas. "Comparative pharmacokinetics of three SGLT-2 inhibitors sergliflozin, remogliflozin and ertugliflozin: an overview." Xenobiotica 47.11 (2017): 1015-1026.

6. Garcia-Ropero, Alvaro, Juan J. Badimon, and Carlos G. Santos-Gallego. "The pharmacokinetics and pharmacodynamics of SGLT2 inhibitors for type 2 diabetes mellitus: the latest developments." Expert Opinion on Drug Metabolism \& Toxicology 14.12 (2018): 1287-1302.

7. American Diabetes Association. "9. Pharmacologic approaches to glycemic treatment: Standards of Medical Care in Diabetes-2021." Diabetes Care 44.Supplement 1 (2021): S111-S124.

8. Gorboulev V, Schurmann A, Vallon V et al (2012) Na+-D-glucose cotransporter SGLT1 is pivotal for intestinal glucose absorption and glucose-dependent incretin secretion. Diabetes 61:187-196

9. Song P, Onishi A, Koepsell H, Vallon V (2016) Sodium glucose cotransporter SGLT1 as a therapeutic target in diabetes mellitus. Expert Opin Ther Targets 20:1109-1125

10. Rieg, Timo, and Volker Vallon. "Development of SGLT1 and SGLT2 inhibitors." Diabetologia 61.10 (2018): 2079-2086.

11. Wiviott, Stephen D., et al. "Dapagliflozin and cardiovascular outcomes in type 2 diabetes." New England Journal of Medicine 380.4 (2019): 347-357.

12. Hsia, Daniel S., Owen Grove, and William T. Cefalu. "An update on SGLT2 inhibitors for the treatment of diabetes mellitus." Current opinion in endocrinology, diabetes, and obesity 24.1 (2017): 73.

13. Nespoux, Josselin, and Volker Vallon. "SGLT2 inhibition and kidney protection." Clinical Science 132.12 (2018): 1329-1339.

14. Zelniker, Thomas A., et al. "SGLT2 inhibitors for primary and secondary prevention of cardiovascular and renal outcomes in type 2 diabetes: a systematic review and meta-analysis of cardiovascular outcome trials." The Lancet 393.10166 (2019): 31-39.

15. Garcia-Ropero, Alvaro, Juan J. Badimon, and Carlos G. Santos-Gallego. "The pharmacokinetics and pharmacodynamics of SGLT2 inhibitors for type 2 diabetes mellitus: the latest developments." Expert Opinion on Drug Metabolism \& Toxicology 14.12 (2018): 1287-1302.

16. Goldenberg, Ronald M., et al. "SGLT2 inhibitor-associated diabetic ketoacidosis: clinical review and recommendations for prevention and diagnosis." Clinical Therapeutics 38.12 (2016): 2654-2664.

17. Kim, Ju-Hyun, et al. "In Vitro Metabolism of DWP16001, a Novel Sodium-Glucose Cotransporter 2 Inhibitor, in Human and Animal Hepatocytes." Pharmaceutics 12.9 (2020): 865.

18. Choi, Min-Koo, et al. "Comparative Pharmacokinetics and Pharmacodynamics of a Novel SodiumGlucose Cotransporter 2 Inhibitor, DWP16001, with Dapagliflozin and Ipragliflozin." Pharmaceutics 
12.3 (2020): 268.

19. Rieg, Timo, et al. "Increase in SGLT1-mediated transport explains renal glucose reabsorption during genetic and pharmacological SGLT2 inhibition in euglycemia." American Journal of Physiology-Renal Physiology 306.2 (2014): F188-F193.

20. Lu, Yasong, et al. "Use of systems pharmacology modeling to elucidate the operating characteristics of SGLT1 and SGLT2 in renal glucose reabsorption in humans." Frontiers in pharmacology 5 (2014): 274.

21. Chen, Xia, et al. "Pharmacokinetics, pharmacodynamics, and safety of single-dose canagliflozin in healthy Chinese subjects." Clinical therapeutics 37.7 (2015): 1483-1492.

22. Komoroski, B., et al. "Dapagliflozin, a novel SGLT2 inhibitor, induces dose-dependent glucosuria in healthy subjects." Clinical Pharmacology \& Therapeutics 85.5 (2009): 520-526.

23. Sha, Sue, et al. "Pharmacodynamic effects of canagliflozin, a sodium glucose co-transporter 2 inhibitor, from a randomized study in patients with type 2 diabetes." PloS one 9.8 (2014): e105638.

24. Kasichayanula, S., et al. "Pharmacokinetics and pharmacodynamics of dapagliflozin, a novel selective inhibitor of sodium-glucose co-transporter type 2, in Japanese subjects without and with type 2 diabetes mellitus." Diabetes, Obesity and Metabolism 13.4 (2011): 357-365.

25. Gilbert, Richard E., and Kevin E. Thorpe. "Acute kidney injury with sodium-glucose co-transporter-2 inhibitors: A meta-analysis of cardiovascular outcome trials." Diabetes, Obesity and Metabolism 21.8 (2019): 1996-2000.

26. Sugiyama, Seigo, et al. "Impact of dapagliflozin therapy on renal protection and kidney morphology in patients with uncontrolled type 2 diabetes mellitus." Journal of clinical medicine research 10.6 (2018): 466.

27. Satirapoj, Bancha, Pattharamon Korkiatpitak, and Ouppatham Supasyndh. "Effect of sodium-glucose cotransporter 2 inhibitor on proximal tubular function and injury in patients with type 2 diabetes: a randomized controlled trial." Clinical kidney journal 12.3 (2019): 326-332.

28. Heyman, Samuel N., et al. "Potential hypoxic renal injury in patients with diabetes on SGLT2 inhibitors: caution regarding concomitant use of NSAIDs and iodinated contrast media." Diabetes Care 40.4 (2017): e40-e41.

29. Szalat, Auryan, et al. "Can SGLT2 inhibitors cause acute renal failure? Plausible role for altered glomerular hemodynamics and medullary hypoxia." Drug safety 41.3 (2018): 239-252.

30. van Meer, Leonie, et al. "Renal effects of antisense-mediated inhibition of SGLT2." Journal of Pharmacology and Experimental Therapeutics 359.2 (2016): 280-289.

31. Kadokura, Takeshi, et al. "Ipragliflozin (ASP1941), a selective sodium-dependent glucose cotransporter 2 inhibitor, safely stimulates urinary glucose excretion without inducing hypoglycemia in healthy Japanese subjects." Diabetology International 2.4 (2011): 172-182.

32. Chaplin, Steve. "SGLT2 inhibitors and risk of genitourinary infections." Prescriber 27.12 (2016): 26-30

\section{Legend to figures}

Figure 1. Mean urinary glucose excretion-time profiles after a single or multiple oral administration of DWP16001 or dapagliflozin. (A) single-dose, B)multiple-doses)

Figure 2. Mean (SD) urinary glucose excretion over 24 hours after single and multiple oral administration of DWP16001 (A) single-dose, B)multiple-doses)

Figure 3. Mean plasma concentration-time profiles after single and multiple oral administration of DWP16001. (A) single-dose, B)multiple-doses)

Figure 4. Relationship between $\mathrm{AUC}_{\text {tau }}$ of DWP16001 after a single dose (from both the single-dose arm \& multiple-dose arm) and urinary glucose excretion over $24 \mathrm{~h}$ after first administration $\left(\mathrm{UGE}_{0-24 \mathrm{~h}}\right)$ 
Figure 5. a) Mean plasma DWP16001 concentration-time profiles and b) cumulative UGE after a single administration at fasted or fed state.

\section{Supplementary appendix}

Figure S1. a)Mean serum glucose and b)insulin profiles before and after multiple oral administration of DWP16001.

\section{Hosted file}

DWP16001_CFT_bjcp.docx available at https://authorea.com/users/448298/articles/547105-dosedependent-glucosuria-of-dwp16001-a-novel-selective-sglt-2-inhibitor-in-healthy-subjects 